\title{
Mechanical and Thermal Properties of Clay- poly[norbornene] Nanocomposites from Ruthenium Alkylidene-mediated Surface-initiated Polymerization
}

\author{
David P. Penaloza Jr.* \\ Department of Chemical Engineering, College of Engineering, Keimyung University, Daegu, Republic of Korea
}

\begin{abstract}
In this work, the mechanical and thermal properties of nanocomposite films prepared from the surface-initiated polymerization of norbornene mediated by a surface-bound ruthenium alkylidine catalyst were investigated. Thermal gravimetric and dynamic mechanical analyses revealed enhanced properties of the resulting nanocomposite films over that of the unfilled polymer.
\end{abstract}

Keywords: polymer nanocomposite; surface-initiated polymerization; metal-mediated ring-opening metathesis polymerization

\section{INTRODUCTION}

The silicate clay-based polymer nanocomposites derived from the use of an organically modified clay montmorillonite (MMT) mineral and other related materials have attracted a great deal of technological and scientific interest owing to the promise of greatly improved properties over those of the unfilled polymer. In incorporating a modified clay to a bulk polymer, several properties - e.g. mechanical strength, thermal stability and flammability and barrier properties - of the polymer-clay nanocomposites are enhanced compared to their neat polymer counterparts (Fornes et al., 2002; Lan et al., 1995; Vaia et al., 1999; Wang et al., 2003; Zanetti et al., 2004).

In order to improve mechanical properties of polymers, fillers in the form of fibers, particles or platelets are incorporated in the polymer matrix to form a composite. The researchers from the Toyota's Central Research and Development Laboratories were the first to demonstrate in the 1980s that a substantial increase in the mechanical properties of a polyamide resin can be realized through reinforcing the nylon matrix with a silicate clay. They successfully prepared a nylon-clay hybrid $(\mathrm{NCH})$ that possessed improved mechanical and thermal properties - a higher modulus, an enhanced strength and a better heat distortion temperature - over the unfilled nylon (Kojima et al., 1994; Usuki et al., 1997; Usuki et al., 1995; Usuki et al., 1995; Yano et al., 1997; Yano et al., 1993).

In this paper, the mechanical and thermal characteristics of clay-based polymer nanocomposite films prepared by extending beyond the incorporation of an organoclay in the polymer matrix by directly growing the

\footnotetext{
*Author to whom correspondence should be addressed; email: david.penaloza.jr@dlsu.edu.ph;

Present address: Department of Chemistry, College of Science, De La Salle University, Manila, Philippines
} 


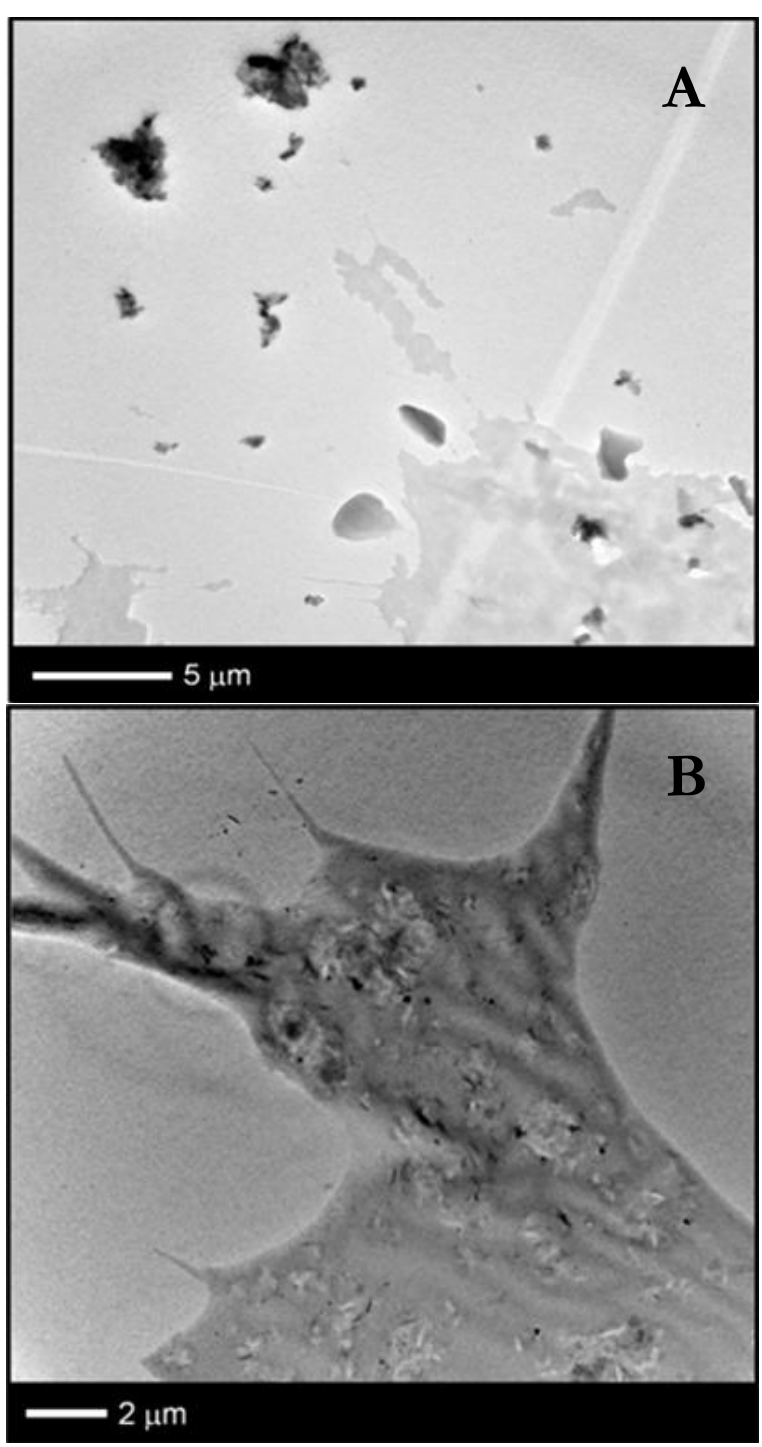

Figure 1. TEM images of (A) a physical mixture of Cloisite $15 A$ clay and poly(norbornene). The scale bar is equivalent to 5 um.; (B) Silylated Cloisite15A claypoly(norbornene) nanocomposite. This is a lower magnified version of Figure $7 B$. The scale bar is equivalent to $2 \mu \mathrm{m}$. (Reproduced from the Polymer Engineering and Science Journal (Penaloza et al., 20157).

polymer structure on the clay surface resulting in covalently tethered long chains. The synthetic route and characterization of the nanocomposites prepared from this metholodogy was previously published (Penaloza et al., 2015). In our previous work, not only a stable attachment between the polymer and the clay could be achieved but having relatively longer polymer chains (as compared to alkyl chains from ammonium modifiers commonly used in clay additives for nanocomposite preparation) increases the possibility of clay structure delamination (exfoliation) (Fan et al., 2002; Fan et al., 2002; Zhou et al., 2001). Here, a surface-attached ruthenium alkylidine catalyst was used to mediate the norbornene polymerization directly from an organoclay surface resulting in nanocomposites where the polymer chains are grafted to the clay by covalent attachment as shown in Figure 1. Two samples for TEM observation were made. The first sample is a physical mixture of the clay template and the polymer where a phase separation was observed. This was completely different from the TEM micrograph of the nanocomposite where the silicate clay platelets can be
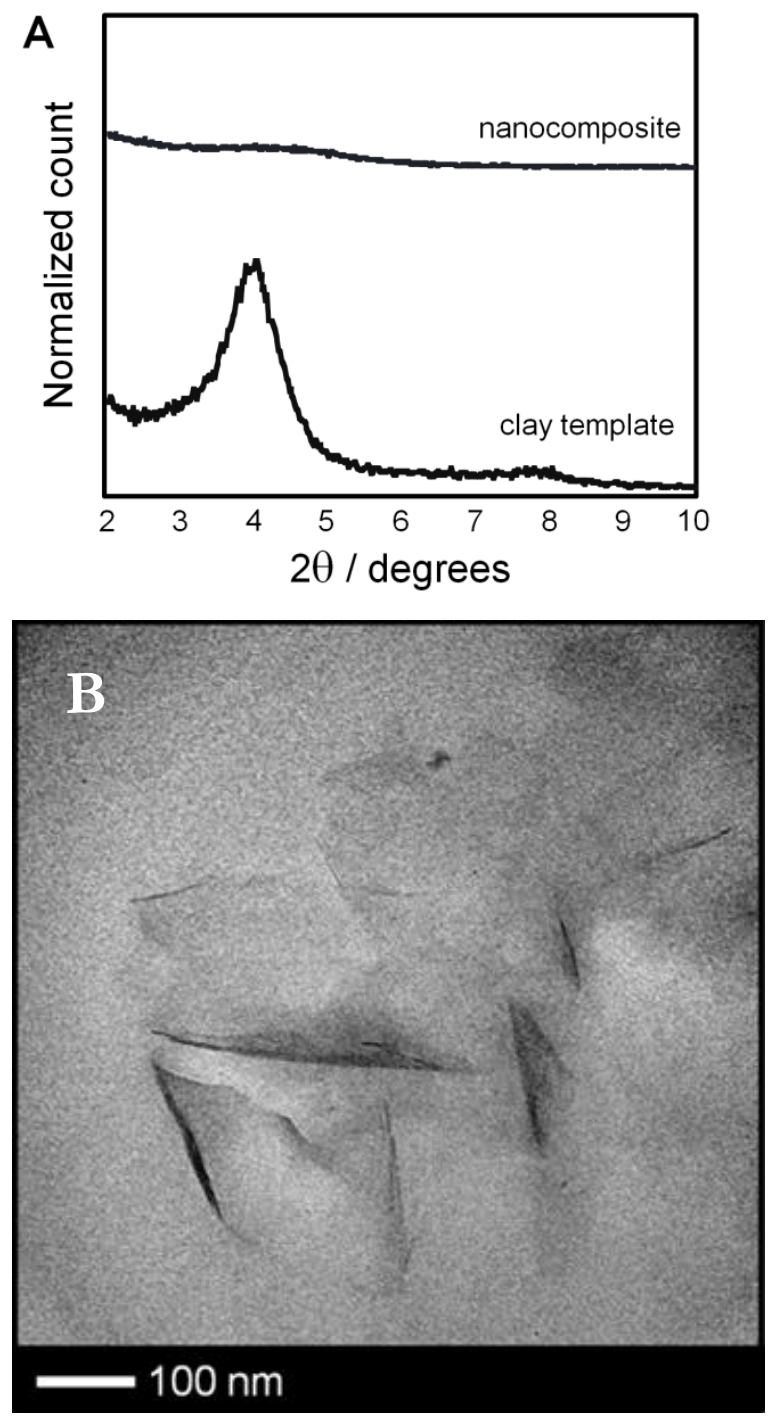

Figure 2. XRD patterns of the silylated Cloisite $15 \mathrm{~A}$ clay before (clay template) and after (nanocomposite) the SI-ROMP of norbornene; (B) TEM micrograph of the nanocomposite. The scale bar is equivalent to $100 \mathrm{~nm}$. (Reproduced from the Polymer Engineering and Science Journal [Penaloza et al., 2015]). 
observed homogeneously dispersed within the boundaries of the polymer matrix (Figure 8B). This confirms that the polymer chains are covalently attached to the clay.

This approach also produced an exfoliated nanocomposite as confirmed by TEM and XRD measurements as shown in Figure 2. (Penaloza et al., 2015). The details of this approach have been published elsewhere (Ahn et al., 2008; Jordi \& Seery, 2005; Penaloza \& Seery, 2009; Yavuz et al., 2009). Such surface-initiated polymerization approach, allows for better control of polymer growth since polymerization starts from immobilized initiators and monomer adds to the propagating polymer chain (Advincula, 2006). The nanocomposites which were then hot-pressed into thin films were prepared from this approach and in this work, their mechanical and thermal properties were compared to that of the free poly(norbornene).

\section{MATERIALS AND METHODS}

\section{Synthesis of clay-poly(norbornene) nanocomposites and free poly(norbornene).}

Synthesis of clay-poly(norbornene) nanocomposites. The procedure described below has been published elsewhere (Penaloza, Seery, 2011; Penaloza \& Seery, 2009; Seery \& Penaloza, 2013; Penaloza et al., 2015). A $100 \mathrm{mg}$ C15Asil was placed in $20-\mathrm{mL}$ scintillation vial and was brought inside glovebox. Five $\mathrm{mL}$ of distilled tetrahydrofuran (THF) - stabilized with TCP, $0.69 \mathrm{~g}$ tricyclohexyl phosphine (TCP) per 150 $\mathrm{mL}$ THF - was used to disperse the clay. Five $\mathrm{mg}$ of Grubbs 1st generation catalyst, a ruthenium alkylidene, was then added to the clay solution and stirred for 5 minutes. The clay solution was then washed with $15 \mathrm{~mL}$ hexane to get rid of unbound catalyst, sealed with parafilm wax and brought outside the glovebox for centrifugation. After centrifugation, clay solution was brought back inside the glovebox, and the supernatant was pipetted off. Then the clay was dispersed in 5 $\mathrm{mL}$ THF. Washing with hexane was done two more times. After the last wash, the clay was re-dispersed in $5 \mathrm{~mL}$ of THF.

In a separate vial, $500 \mathrm{mg}$ of norbornene monomer was dissolved in $10 \mathrm{~mL}$ of THF (with TCP). Then the monomer and the activated clay solutions were combined and allowed to stir for the desired reaction times.

Two sets of clay-poly(norbornene) nanocomposites were prepared using 120 and 180 minutes of polymerization time, respectively. For each reaction time, 5 replicates were used to produce enough materials for film preparation. For each replicate, the reaction was terminated by adding $0.5 \mathrm{~mL}$ of ethyl vinyl ether (EVE). The mixture was then brought out of the glovebox and precipitated in cold methanol. The polymer nanocomposites were collected by gravity filtration and dried under vacuum. The final composition of the clay and the polymer in each of the nanocomposite was determined by thermogravimetric analysis (TGA).

Synthesis of free poly(norbornene). The free poly(norbornene) sample was prepared in glovebox. In a $20-\mathrm{mL}$ scintillation vial, $500 \mathrm{mg}$ of norbornene monomer was dissolved in 10 $\mathrm{mL}$ of THF. $5 \mathrm{mg}$ of the Grubbs $1 \mathrm{st}$ generation catalyst was then added to the monomer solution. The mixture was allowed to stir for the 120 minutes. Five replicates were used to produce enough materials for film preparation. The reaction was terminated by adding $0.5 \mathrm{~mL}$ of EVE. The mixture was then brought out of the glovebox and precipitated in cold methanol. The polymer was collected by gravity filtration and dried under vacuum.

Physical property measurements of claypoly(norbornene) nanocomposites and free poly(norbornene). Dynamic mechanical analysis (DMA). To compare the mechanical properties of the nanocomposites from the unfilled poly(norbornene), a dynamic mechanical analysis (DMA) was performed. The storage and loss moduli of the poly(norbornene) and the clay-poly(norbornene) nanocomposite samples were obtained by DMA, at a heating rate of $5{ }^{\circ} \mathrm{C} / \mathrm{min}$ from $50{ }^{\circ} \mathrm{C}$ to $150{ }^{\circ} \mathrm{C}$. Also, a stress-strain testing was also conducted on the unfilled poly(norbornene) films and two claypoly(norbornene) nanocomposites which were determined to contain $5 \%$ (C15Asil_PNB5) 
and $13 \%$ organoclay (C15Asil_PNB13). Three replicates for each of the sample films were used. From the stress-strain plot, the tensile modulus of the samples was determined using the TA Instruments Universal Analysis 2000 software.

Thermogravimetric Analysis (TGA). Thermogravimetric analysis was performed to determine the thermal properties of the organoclay, poly(norbornene) and the nanocomposites that include degradation temperature $\left(\mathrm{T}_{\mathrm{d}}\right)$ and total residual mass at $1000{ }^{\circ} \mathrm{C}\left(\mathrm{m}_{1000}{ }^{\circ} \mathrm{C}\right)$. Samples were placed in alumina pan and were heated up to $1000{ }^{\circ} \mathrm{C}$ under nitrogen atmosphere with a heating rate of $10{ }^{\circ} \mathrm{C} / \mathrm{min}$. TGA analyses of the clay, polymer and clay-polymer nanocomposites were carried out on a TA Instruments SDT Q600 Simultaneous TGA/DSC. TA Instruments Universal Analysis 2000 software was used to process the data.

\section{RESULTS AND DISCUSSIONS}

Mechanical properties of the claypoly(norbornene) nanocomposites and the free poly(norbornene). To study the effect of the clay composition in the mechanical properties of the nanocomposites prepared by surface initiated ring opening metathesis polymerization (SI-ROMP), sample films of poly(norbornene) and clay-poly(norbornene) nanocomposites containing $5 \%$ (C15Asil_PNB5) and $13 \%$ organoclay (C15Asil_PNB13) (as determined by TGA) were prepared using hot press. The stressstrain test was performed on these samples using dynamic mechanical analysis (DMA). Three replicates for every film sample were used. The tensile modulus of the different samples was determined as the slope of the stress-strain plot (Figure 3).

The calculated values of the tensile modulus of the different poly(norbornene) films are presented in Figure 3. Both nanocomposite samples have higher tensile moduli than the unfilled poly(norbornene) sample. The nanocomposite containing $5 \%$ organoclay (C15Asil_PNB5) registers a tensile modulus equal to $675 \pm 26 \mathrm{MPa}$ (85\% increase) while

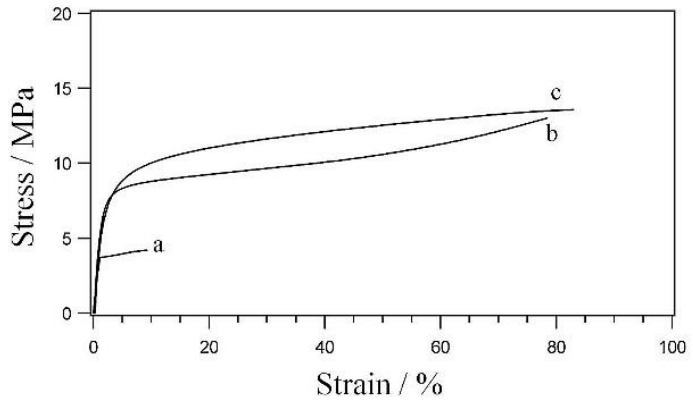

Figure 3. Stress-strain plot the poly(norbornene) films containing varying amounts of C15Asil organoclay: (a) $0 \%$; (b) $5 \%$ and (c) $13 \%$.

$13 \%$ organoclay (C15Asil_PNB13) increased the film modulus to $833 \pm 23 \mathrm{MPa}(128 \%)$. Without the organoclay filler, the poly(norbornene) film samples have an average tensile modulus of only $365 \pm 23$ $\mathrm{MPa}$. Also, both nanocomposite samples have exhibited considerably higher percent elongation than the pure poly(norbornene) (Figure 3).

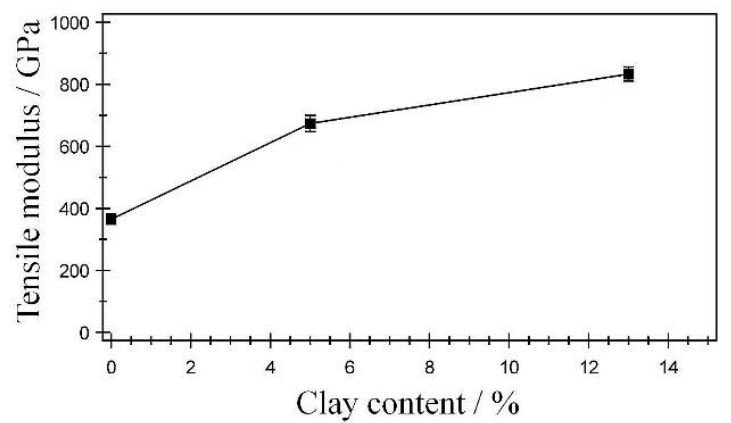

Figure 4. Tensile moduli of the poly(norbornene) films containing varying amounts of C15Asil organoclay: (a) $0 \%$; (b) $5 \%$ and (c) $13 \%$.

Figures $5 \mathrm{a}-\mathrm{b}$ present the loss and storage modulus of the poly(norbornene) and the clay-poly(norbornene) nanocomposite. Sample films were run from $-50{ }^{\circ} \mathrm{C}$ to $150{ }^{\circ} \mathrm{C}$. DMA measurements show that the nanocomposite sample has higher storage and loss modulus as compared to the unfilled poly(norbornene) film.

Several studies in the literature have attributed the increase in mechanical properties of the nanocomposites over the unfilled polymer due to the homogeneous dispersion of the inorganic fillers in the bulk material 

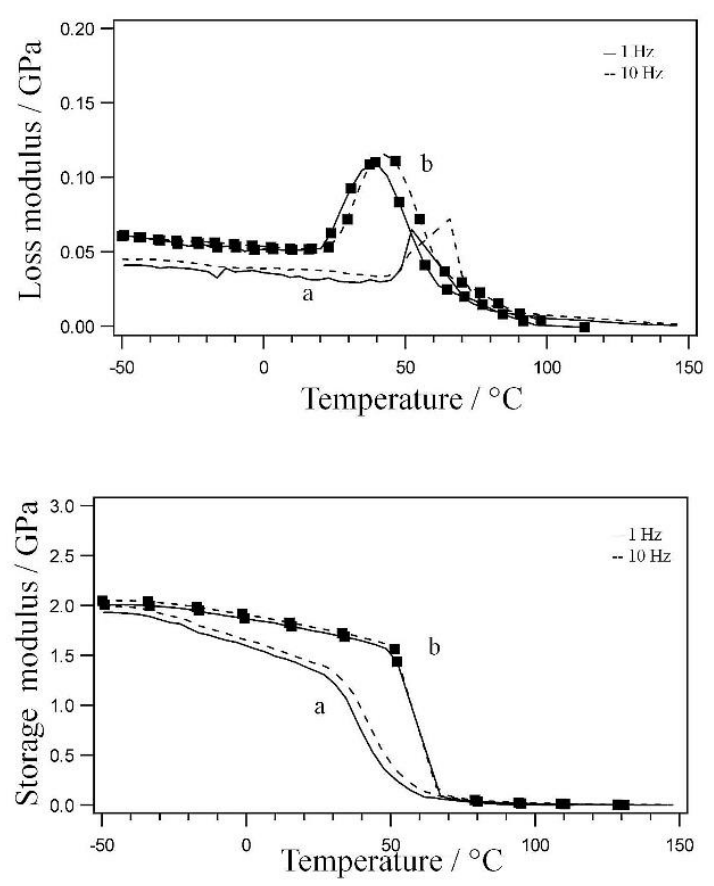

Figure 5. Loss and storage moduli of (a) free poly(norbornene) film and (b) C15Asilpoly(norbornene) nanocomposite film (13\% clay).

(Alexandre \& Dubois, 2000; Awad et al., 2009; Braganca et al., 2007; Kawasumi et al., 1997; Kaynak et al., 2009; Maiti et al., 2002; Pramanik et al., 2003; Ray et al., 2003). The stable grafting of the polymer chains to the inorganic clay substrate may have also contributed to the increased tensile modulus of the clay-filled poly(norbornene) films. For instance, in the work of Karesoja et al. (2009) on the preparation of a clay-poly(butyl acrylate-comethyl methacrylate) nanocomposite via SIATRP, the mechanical properties of the polymer films were improved as a function of the organoclay content. The authors also observed that the polymer-modified clay when used as fillers affects the stress-strain properties more than using only unmodified MMT clay. In their work, they grafted the poly(butyl acrylate-co-methyl methacrylate) on the MMT organoclay and use this polymermodified clay as a filler in the same poly(butyl acrylate-co-methyl methacrylate) copolymer as the matrix.

Thermal properties of the claypoly(norbornene) nanocomposites and the free poly(norbornene). Thermogravimetric analysis (TGA). The thermal stability of the organomodified clay plays a key role in the preparation and processing of clay-polymer nanocomposites. In this study, a comparison of the thermal properties of the organoclay, poly(norbornene) and clay-poly(norbornene) nanocomposites was made using thermogravimetric (TGA) measurements. The thermal properties considered in this study include the degradation temperature and the total residual mass after heating the samples to $1000{ }^{\circ} \mathrm{C} \quad\left(\mathrm{m}_{1000^{\circ}} \mathrm{C}\right)$. These properties are summarized in Table 1.

Table 1. Thermal Properties of the C15Asil Organoclay, Poly(norbornene) and Claypoly(norbornene) Nanocomposites.

\begin{tabular}{|c|c|c|}
\hline Samples & $\mathbf{T}_{\mathrm{d}}$ & $\mathrm{m}_{1000{ }^{\circ} \mathrm{C}}$ \\
\hline $\mathrm{C} 15 \mathrm{Asil}^{\mathrm{a}}$ & $300{ }^{\circ} \mathrm{C}$ & $59 \%$ \\
\hline Poly(norbornene) ${ }^{\mathrm{b}}$ & $453^{\circ} \mathrm{C}$ & $0 \%$ \\
\hline \multicolumn{3}{|c|}{ Clay-poly(norbornene) nanocomposite } \\
\hline C15Asil_PNB13c $^{c}$ & $462^{\circ} \mathrm{C}$ & $8 \%$ \\
\hline C15Asil_PNB5 $^{c}$ & $462{ }^{\circ} \mathrm{C}$ & $3 \%$ \\
\hline
\end{tabular}

The clay-poly(norbornene) nanocomposite samples degrade at a much higher temperature $\left(462^{\circ} \mathrm{C}\right)$. The degradation temperature for the unfilled poly(norbornene) is $453^{\circ} \mathrm{C}$.

Another point of comparison is between the C15Asil (organoclay only) and the nanocomposites. Two kinds of organoclays can be considered in this comparison: (1) the C15Asil as an organoclay that is modified with alkyl ammonium salt via an ion exchange reaction and (2) the clay-poly(norbornene) nanocomposites as organoclays modified by polymer chains via surface-initiated ROMP. The nanocomposites (as a polymer-modified organoclay) exhibit a much higher $T_{d}$ as compared to the C15Asil indicating better thermal properties of the nanocomposites. The observed difference between the two kinds of organoclays can be attributed to the stability of the chain attachment - with the nanocomposites having chains attached by covalent bonding as compared to the C15Asil having alkyl chains tethered by ionic attachment. 


\section{CONCLUSIONS}

The clay-poly(norbornene) nanocomposites exhibited better mechanical properties and higher thermal stability as compared to the unmodified poly(norbornene). DMA studies showed that the clay reinforcement increases the tensile, storage and loss moduli as well as percent elongation of the nanocomposites. TGA measurements, on the other hand, confirmed that the nanocomposites were found to degrade at a much higher temperature compared to the free poly(norbornene).

\section{ACKNOWLEDGMENT}

This research was supported by the Bisa Research Grant of Keimyung University in 2013. Prof. Thomas AP Seery of the University of Connecticut is also acknowledged.

\section{REFERENCES}

Advincula RC. Polymer brushes by anionic and cationic surface-initiated polymerization (SIP). Surface-Initiated Polymerization I (Vol. 197, pp. 107-136). Berlin: Springer-Verlag Berlin; 2006.

Ahn SK, Penaloza DP, Sandberd DJ, Kasi R, Seery TAP. Nanocomposites from silica nanoparticles poly(norbornenes) with pendant cholesterols. Polym. Prep. (Am. Chem. Soc., Div. Polym. Chem.) 2008; 49:760-761.

Alexandre M, Dubois P. Polymer-layered silicate nanocomposites: preparation, properties and uses of a new class of materials. Mater. Sci. Eng. R-Rep. 2000; 28: 1-63.

Awad WH, Beyer G, Berderly D, Ijdo WL, Songtipya P, Jimenez-Gaso MD. Material properties of nanoclay PVC composites. Polymer. 2009; 50: 1857-1867.

Braganca FDC, Valadares LF, Leite CADP, Galembeck F. Counterion effect on the morphological and mechanical properties of polymer-clay nanocomposites prepared in an aqueous medium. Chem.Mat. 2007; 19:33343342.
Fan X, Xia C, Fulgum T, Park MK, Locklin J, Advincula RC. Polymer brushes grafted from clay nanoparticles adsorbed on a planar substrate by free radical surface-initiated polymerization. Langmuir. 2002; 19:916-923.

Fan X, Zhou Q, Xia C, Cristofoli W, Mays J, Advincula RC. Living anionic surface-initiated polymerization (LASIP) of styrene from clay nanoparticles using surface bound 1,1diphenylethylene (DPE) initiators. Langmuir. 2002; 18:4511-4518.

Fornes TD, Yoon PJ, Hunter DL, Keskkula H, Paul DR. Effect of organoclay structure on nylon 6 nanocomposite morphology and properties. Polymer. 2002; 43:5915-5933.

Jordi MA, Seery TAP. Quantitative determination of the chemical composition of silica-poly(norbornene) nanocomposites. J. Am. Chem. Soc. 2005; 127:4416-4422.

Karejosa M, Jokinen H, Karkalainen E, Pulkkinen P, Torkkeli M, Soininen A. Grafting of montmorillonite nano-clay with butyl acrylate and methyl methacrylate by atom transfer radical polymerization: Blends with poly(BuA-co-MMA). J. Polym. Sci. A Polym. Chem. 2009; 47:3086-3097.

Kawasumi M, Hasegawa N, Kato M, Usuki A, Okada A. Preparation and mechanical properties of polypropylene-clay hybrids. Macromolecules. 1997; 30:6333-6338.

Kaynak C, Nakas GI, Isitman NA. Mechanical properties, flammability and char morphology of epoxy resin/montmorillonite nanocomposites. Appl. Clay Sci. 2009; 46:319-324.

Kojima Y, Usuki A, Kasumi M, Okada A, Kurauchi T, Kamigaito O. Fine-structure of nylon-6-clay hybrid. J. Polym. Sci. Pol. Phys. 1994; 32:625-630.

Lan T, Kaviratna PD, Pinnavaia TJ. Mechanism of clay tactoid exfoliation in epoxy-clay nanocomposites. Chem. Mat. 1995; 7:2144-2150. 
Maiti P, Nam PH, Okamoto M, Hasegawa N, Usuki A. Influence of crystallization on intercalation, morphology, and mechanical properties of polypropylene/clay nanocomposites. Macromolecules. 2002; 35:2042-2049.

Penaloza DP. Synthesis and characterization of polymer-clay systems prepared by surfaceinitiated polymerization [dissertation]. [Connecticut, USA]: University of Connecticut; 2011. 244p.

Penaloza DP, Sandberg D, Giotto MV, Seery TAP. An exfoliated clay-poly(norbornene) nanocomposite prepared by metal-mediated surface-initiated polymerization. Polym. Eng. Sci. 2015; 55: 2349-2354.

Penaloza DP, Seery TAP. Synthesis and characterization of montmorillonitepoly(norbornene) nanocomposite. Polym. Prep. (Am. Chem. Soc., Div. Polym. Chem.) 2009; 50:655-656.

Pramanik M, Srivasta SK, Samantary BK, Bhowmick AK. EVA/Clay nanocomposite by solution blending: Effect of aluminosilicate layers on mechanical and thermal properties. Macromol. Res. 2003; 11:260-266.

Ray SS, Okamoto K, Okamoto M. Structureproperty relationship in biodegradable poly(butylene succinate)/layered silicate nanocomposites. Macromolecules. 2003; 6:2355-2367.

Seery TAP, Penaloza DP. Montmorillonite poly(norbornene) nanocomposites from ruthenium alkylidene surface-initiated polymerization. Proceedings of the 245th American Chemical Society (ACS) National Meeting and Exposition; 2013 April 7-11; Louisiana, USA: American Chemical Society. 2013; p. POLY53.

Usuki A, Kato M, Okada A, Kurauchi T. Synthesis of polypropylene-clay hybrid. J. Appl. Polym. Sci. 1997; 63:137-138.
Usuki A, Kawasumi M, Kojima Y, Okada A, Kurauchi T. Synthesis and properties of diamine-modified nylon 6-clay hybrid. Kobunshi Ronbunshu. 1995; 52:440-444.

Usuki A, Koiwai A, Kojima Y, Kawasumi M, Okada A, Kurauchi T. Interaction of nylon-6 clay surface and mechanical properties of nylon-6 clay hybrid. J. Appl. Polym. Sci. 1995; 55:119-123.

Vaia RA, Price G, Ruth PN, Nguyen HT, Lichtenhan J. Polymer/layered silicate nanocomposites as high performance ablative materials. Appl. Clay Sci. 1999; 15:67-92.

Wang S, Hu Y, Lin Z, Gui Z, Wang Z, Chen Z. Flammability and thermal stability studies of ABS/montmorillonite nanocomposite. Polym. Int. 2003; 52:1045-1049.

Yano K, Usuki A, Okada A. Synthesis and properties of polyimide-clay hybrid films. J. Polym. Sci. A Polym. Chem. 1997; 35:2289-2294.

Yano K, Usuki A, Okada A, Kurachi T, Kamigaito O. Synthesis and properties of polyimide-clay hybrid. J. Polym. Sci. A Polym. Chem. 1993; 31:2493-2498.

Yavuz MS, Jensen GC, Penaloza DP, Seery TAP, Pendergraph SA, Rusling JF. Gold nanoparticles with externally controlled, reversible shifts of local surface plasmon resonance bands. Langmuir. 2009; 25:13120-13124.

Zanetti M, Bracco P, Costa L. Thermal degradation behaviour of PE/clay nanocomposites. Polym. Degrad. Stabil. 2004; 85:657-665.

Zhou Q, Fan X, Xia C, Mays J, Advincula RC. Living anionic surface initiated polymerization (SIP) of styrene from clay surfaces. Chem. Mat. 2001; 13:2465-2467. 